\title{
BASIC GROUPS OF LIE ALGEBRAS AND HOPF ALGEBRAS
}

\begin{abstract}
NAZIH NAHLUS
Let $L$ be a finite-dimensional Lie algebra over an algebraically closed field $F$ of characteristic 0 , let $H(L)$ be the Hopf algebra of representative functions of $L$, and let $B(L)$ be the Hochschild basic group $B(L)$ of $L$.

By using Hochschild theory of $H(L)$, we show that two such Lie algebras have the same Hopf algebra if and only if they have the same basic group, or equivalently, they have the same basic Lie algebra (the Lie algebra of the basic group). This is shown by first obtaining the following characterization of $B(L)$. If $G(L)$ is the pro-affine algebraic group associated with $H(L)$, then $B(L)$ is the quotient of $G(L)$ by the intersection of the radical of $G(L)$ with the reductive part of the center of $G(L)$. We also show that the basic Lie algebra of $L$ can be constructed, up to isomorphism, directly from the adjoint representation of $L$.

Finally, we apply the theory of basic groups to obtain an intrinsic characterization of the Hopf algebras (over $F$ ) that are isomorphic to $H(L)$ for some Lie algebra $L$.

Some applications to algebraic Lie algebras are also considered.
\end{abstract}

\section{Introduction.}

Let $L$ be a finite-dimensional Lie algebra over an algebraically closed field $F$ of characteristic 0 . Then the Hopf algebra $H(L)$ of representative functions of $L$ is the (continuous) dual of the universal enveloping algebra $U(L)$ of $L$ whose elements are the linear functionals on $U(L)$ which vanish on an ideal of finite codimension $[\mathbf{H 4}$, p. 56], [S]. The Hopf algebra $H(L)$ over $F$ has been investigated by Hochschild [H2] - [H6]. If $L=[L, L]$, then $L$ is canonically isomorphic to the Lie algebra of all differentiations of $H(L)$ [H4, Thm. 6.1]. But, in general, there exists an uncountable collection of non-isomorphic Lie algebras with isomorphic Hopf algebras [H-M5, p. 1150]. However, $H(L)$ can still determine many invariants of $L$ of which the most important seems to be the Hochschild basic group $B(L)$ of $L$ which is a naturally defined affine algebraic group whose Lie algebra contains $L$ as an algebraically dense Lie algebra [H6, Section 3]. 
More specifically, $B(L)$ is the affine algebraic group corresponding to the smallest regular Hopf subalgebra of $H(L)$ (see preliminaries).

Over the complex field $\mathbb{C}$, Magid has investigated $H(L)$ by introducing the notion of the bottom group of $L$ (or, of $G(L)$ ). This is it $\operatorname{Bot}(L)=$ $G(L) / Q(G(L))$ where $G(L)$ is the pro-affine algebraic group associated with $H(L)$, and $Q(G(L))$ is the intersection of the radical of $G(L)$ with the reductive part of the center of $G(L)$. One of Magid's results is that $\operatorname{Bot}(L)$ determines $H(L)$ up to isomorphism, so that $H(L)$ determines $B o t(L)$ and vice versa [Ma3, Thm. 6]. Moreover, $\operatorname{Bot}(L)$ is isomorphic to every reduced regular hull of the simply connected complex analytic group with Lie algebra $L$ [Ma3, Thm. 5]. This last result suggests that $B(L)$ and $B o t(L)$ are equal over $\mathbb{C}$. We are thus interested in the following problems.

Problem 1. Is $B(L)=G(L) / Q(G(L))$ over $F$ ? (i.e., is $B(L)=\operatorname{Bot}(L)$ ?)

Problem 2. Does $H(L)$ determine $B(L)$ and vice versa?

Problem 3. (i) Does $H(L)$ determine the Lie algebra of $B(L)$ and vice versa? (ii) Can we construct the basic Lie algebra of $L$ (i.e., the Lie algebra of $B(L)$ ) directly from the adjoint representation of $L$ ?

Problem 4. Find an intrinsic characterization of the Hopf algebras over $F$ isomorphic to $H(L)$ for some Lie algebra $L$.

Our answers to the above problems are as follows. 1. $B(L)=G(L) / Q(G(L))$ over $F$ (Corollary 2.5). The proof of Theorem 2.3 which implies Corollary 2.5, relies on a translation of Hochschild's main results on $H(L)$ to pro-affine algebraic group terms keeping track of the regular Hopf subalgebras of $H(L)$. Such a translation is done in Theorem 2.2. In Theorem 2.3, we give characterizations in affine terms as well as in pro-affine terms, of the regular hulls of $L$ (corresponding to the regular Hopf sub-algebras of $H(L)$ ). Theorem 2.3 generalizes many known results over $\mathbb{C}$. Moreover, Theorem 2.3 implies Theorem 3.1 of [H6] (see Corollary 2.6). However, its proof is not a routine generalization of the proofs in the complex case. For example, Magid's proof of Theorem 5 in [Ma3] relies on an intrinsic (affine) characterization of the regular algebraic hulls of complex analytic groups established in [H-M3, Thms. 3.1 and 4.1]; but the analogous results over $F$ have not been yet established.

2. $H(L)$ determines $B(L)$ and vice versa (Corollary 4.3 ).

This follows by combining our result $B(L)=G(L) / Q(G(L))$ and an argument of Magid showing that $G(L) / Q(G(L))$ determines $G(L)$ up to isomorphism.

3. (i) $H(L)$ determines $\mathcal{L}(B(L))$ and vice versa (Corollary 4.3).

This follows by combining the preceding result, the fact that $B(L)$ is almost simply connected (Corollary 2.5), and Theorem 3.1 which says that an almost 
simply connected affine algebraic group $G$ is determined up to isomorphism from its Lie algebra $\mathcal{L}(G)$. (Moreover, $G$ is a direct factor of the basic group of $\mathcal{L}(G)$.)

(ii) If ad $: L \rightarrow \operatorname{Der} L$ is the adjoint representation of $L$, and $T$ is a maximal toral subalgebra of the radical of the algebraic hull of ad $(L)$ in DerL, then $L+T$ (semi-direct) with the adjoint action of $T$ on $L$ is isomorphic to the basic Lie algebra $\mathcal{L}(B(L))$ of $L$ (Theorem 4.4).

4. If $A$ is a commutative Hopf algebra over $F$, then $A$ is isomorphic to some Hopf algebra $H(L)$ where $L$ is a finite-dimensional Lie algebra if and only if A satisfies the following properties:

(i) A is an integral domain;

(ii) there is a group isomorphism from the additive group $P$ of the primitive elements of $A$ onto the multiplicative group $Q$ of the group-like elements of $A$;

(iii) there exists a right stable affine subalgebra $B$ of $A$ such that $A=$ $B \otimes F[Q]$, and $B$ has no proper affine unramified extensions (Theorem 5.2).

The proof of this result relies on a generalization of Magid's intrinsic characterization of the affine algebraic groups isomorphic to $\operatorname{Bot}(L)$ for some $L$, as well as his argument that $\operatorname{Bot}(L)$ determines $G(L)$ up to isomorphism [Ma3, Thms. 6 and 13].

We note that our criteria for Hopf algebras isomorphic to $H(L)$ are slightly simpler and sharper than those of Reinoehl in [R2, p. 181] (which are (i) and (ii) as in our criteria above together with the following variation on (iii): there exists a left stable affine subalgebra $B$ of $A$ such that $A=B[Q]$, $A$ is a free $B$-module with basis $Q$, and the semi-simple part $B_{S}$ of $B$ is a Hopf subalgebra of $A$ and has no proper affine unramified extension in any Hopf algebra containing $B_{S}$ ). We would like also to note that our proof is much easier than that of Reinoehl in [R2], although more is shown by Reinoehl if $A$ satisfies his criteria. Note that his result is a generalization of a similar result for complex analytic groups due to Hochschild and Mostow in [H-M5, Thm. 2.1].

We rely on [H2] - [H6] for basic results concerning $H(L)$, and on [H-M3] and $[\mathbf{H 5}]$ for basic results on pro-affine algebraic groups.

Acknowledgements. The author wishes to thank Professor Hochschild for the many enlightening discussions on some parts of this paper.

\section{Preliminaries.}

All Lie algebras, Hopf algebras, and pro-affine algebraic groups are considered over an algebraically closed field $F$ of characteristic 0 , and $L$ is a 
finite-dimensional Lie algebra over $F$. If $G$ is a pro-affine algebraic group, $A[G]$ is its Hopf algebra of polynomial functions, $\operatorname{Hom}(G, F)$ is its group of rational (polynomial) additive characters, $X(G)$ is its group of rational multiplicative characters, $\mathcal{L}(G)$ is its Lie algebra, $G_{1}$ is its identity component, $G_{u}$ is its unipotent $\operatorname{radical}, \operatorname{rad}(G)$ is its radical, and $[G, G]$ is its commutator subgroup. $[L, L]$ and $\operatorname{rad}(L)$ are defined the same way, and $U(L)$ is the universal enveloping algebra of $L$. If $f$ is a morphism of pro-affine algebraic groups, $f^{0}$ is its differential, and ker $f$ is its kernel.

A subspace of $H(L)$ is left (resp. right) stable under the left (resp. right) translation action of $U(L)$ given by $(u . f)(x)=f(x u)$ (resp. $(f . u)(x)=$ $f(u x))$ where $f \in H(L)$ and $u \in U(L)$. If $G$ is a pro-affine algebraic group, a subspace of $A[G]$ is left (resp. right) stable if it is stable under the left (resp. right) translation action of $G$ given by $(g . f)(x)=f(x g)(\operatorname{resp} .(f \cdot g)(x)=$ $f(g x))$ where $g \in G$ and $f \in A[G]$. If $S$ is a subspace of a Hopf algebra $A$ with comultiplication $\gamma$ where $A=H(L)$ or $A=A[G]$, then $S$ is left (resp. right) stable in the above sense if and only if $\gamma(S) \subset S \otimes A$ (resp. $\gamma(S) \subset A \otimes S)$. The subspace $S$ is called bistable if it is both left and right stable.

Let $Q(L)$ be the (multiplicative) group of group-like elements of $H(L)$, and let $P(L)$ be the (additive) group of primitive elements of $H(L)$. Then there is a group isomorphism from $P(L)$ to $Q(L)$ given by the exponential map: if $p \in P(L), \exp (p)$ is the unique algebra homomorphism $U(L) \rightarrow F$ whose restriction to $L$ coincides with $p$ [R2, p. 183]. Note that $P(L)=$ $\operatorname{Hom}(G(L), F)$ and $Q(L)=X(G(L))$ since $H(L)=A[G(L)]$.

The following result is contained in [H2, Thm. 5] and [H3, pp. 610-611].

Theorem 1.1. There exists a subalgebra $B$ of $H(L)$ satisfying the following conditions:

(1) $B$ is a right stable affine (finitely generated) subalgebra.

(2) $H(L)=C \otimes B$ where $C$ is the Hopf subalgebra $F[Q(L)]$. That is the multiplication map is an algebra isomorphism from $C \otimes B$ to $H(L)$. In particular, $B$ separates the elements of $L$.

(3) $B_{S}=H(L)^{R}$ where $R=\operatorname{rad}(L), B_{S}$ is the subalgebra of $B$ consisting of representative functions belonging to semisimple representations of $L$, and $H(L)^{R}$ is the left $R$-annihilated (Hopf subalgebra) of $H(L)$.

(4) $B=H(L)^{R} \otimes V^{*}$ where $V^{*}$ is a right stable subalgebra whose (Krull) dimension is equal to the dimension of the radical $R$ of $L$. Moreover, $P(L) \subset V^{*}$.

A normal basic subalgebra of $H(L)$ is a subalgebra $B$ satisfying (1)-(3) of Theorem 1.1, and hence satisfying (4) because normal basic subalgebras are 
conjugate by an automorphism of $H(L)$ of the $\operatorname{Exp}(t(x))$ where $t$ is the left translation map and $x \in[L, R][\mathbf{G}$, Thm. 4.1].

The basic Hopf subalgebra $B^{*}(L)$ of $H(L)$ is defined as the smallest Hopf subalgebra of $H(L)$ containing some (and hence every) normal basic subalgebra of $H(L)$. Since every normal basic subalgebra is affine, then so is $B^{*}(L)$. Hence $B^{*}(L)$ is the Hopf algebra of polynomial functions on an affine algebraic group $B(L)$, termed the basic group of $L$ [H6, p. 173]. We shall identify $L$ with its cannonical image in $\mathcal{L}(G(L))$, the Lie algebra of all differentiations of $A[G(L)]=H(L)[\mathbf{H 2}$, p. 62]. Moreover $L$ can be also identified with its cannonical image in $\mathcal{L}(B(L))$ because $A[B(L)]$ separates the elements of $L$ by condition (2) of Theorem 1.1 [H6, p. 175].

\section{Remarks.}

1) The normal basic subalgebras of $H(L)$ can be characterized purely in terms of the Hopf algebra $H(L)$. Specifically, the third condition in the definition of a normal basic subalgebra can be replaced with the condition that $B_{S}$ is a Hopf subalgebra of $H(L)$ [G, Thm. 3.1]. Moreover, the semisimple elements of $H(L)$ can be characterized purely in terms of $H(L)$ [R2, p. 180]. So indeed, $H(L)$ determines $B(L)$.

2) $G(L)$ has the following universal property. If $H$ is a connected affine algebraic group (over $F$ ), and $i: L \rightarrow \mathcal{L}(H)$ is a Lie algebra homomorphism whose image is algebraically dense, then there exists a canonical surjective morphism $f: G(L) \rightarrow H$ whose differential agrees with $i$ on L. Moreover, $A[H]$ can be identified with its canonical image in $H(L)$, (so $f$ is a restriction morphism). To see this, let $\pi: A[H] \rightarrow H(\mathcal{L}(H))$ be the natural morphism of Hopf algebras such that $\pi(f)(u)=(u . f)\left(1_{H}\right)$ for every $f \in A[H]$ and $u \in U(\mathcal{L}(H))$ where $A[H]$ is viewed as a left $U(\mathcal{L}(H))$-module via the (proper) derivation action of $\mathcal{L}(H)$ on $A[H]$. Then $\pi$ is injective because $H$ is irreducible over $F[\mathbf{H 1}$, Thm. 3.1, p. 230]. If $\hat{i}: H(\mathcal{L}(H)) \rightarrow H(L)$ is the morphism induced by $i$. Then $\hat{i}$ is injective since $i(L)$ is algebraically dense in $\mathcal{L}(H)$. Hence $A[H]$ can be viewed as a Hopf subalgebra of $H(L)$ via $\hat{i} \circ \pi$. Hence we have a surjective morphism $f: G(L) \rightarrow H$ whose differential agrees with $i$ on $L$.

3) The category $\operatorname{Mod}(L)$ of finite-dimensional $L$-modules, as a tensored abelian category of finite-dimensional $F$-spaces, determines the Hopf algebra $H(L)$ and vice versa. To see this, let $\operatorname{Mod}(G(L))$ be the category of finite-dimensional $G(L)$-modules, as a tensored abelian category of finitedimensional $F$-spaces. Then the universal property of $G(L)$ implies that the category $\operatorname{Mod}(L)$ determines the category $\bmod (G(L))$ and vice versa. The proof of Proposition 2.3 of $[\mathbf{L}]$ implies that the category $\operatorname{Mod}(G(L))$ determines $G(L)$ and vice versa (see also [Ma5, p. 54]). Hence the category 
$\operatorname{Mod}(L)$ determines the Hopf algebra $H(L)$ and vice versa.

\section{Basic groups of Lie algebras.}

We shall need to interpret Theorem 1.1 in pro-affine algebraic group terms. For this, we need the following basic result, but, in the absence of a convenient reference, we give a proof here.

Lemma 2.1. Let $G$ be a pro-affine algebraic group (over $F$ ), and suppose that $A[G]=S \otimes T$ (as algebras) where $S$ is a bistable subalgebra of $A[G]$ and $T$ is a right stable subalgebra. Let $S^{\prime}$ be the fixer of $S$ in $G$ (under left translations), and let $T^{\prime}$ be the fixer of $T$ in $G$. Then $G=S^{\prime} \cdot T^{\prime}$ (semidirect) where $S^{\prime}$ is normal in $G$. Moreover, the $S^{\prime}$-fixed part $A[G]^{S^{\prime}}$ (under left translations) coincides with $S$ and the $T^{\prime}$-fixed part $A[G]^{T^{\prime}}$ coincides with $T$. In particular, $S$ is a Hopf subalgebra of $A[G]$ isomorphic to $A\left[T^{\prime}\right]$ as Hopf algebras, and $T$ is isomorphic to $A\left[S^{\prime}\right]$ as algebras.

Proof. By considering the left translation action of $G$ on $A[G]$, we can view $G$ as the group of proper automorphisms of $A[G]$. These are the automorphisms of $A[G]$ commuting with the right translation action $f \rightarrow f . g$ [H-M4, p. 1128]. Since $S$ is left stable, $S$ is stable under the proper automorphisms of $A[G]$. Consequently $S^{\prime}$ is normal in $G$. Moreover, if $g \in G$, then $g$ restricts to an automorphism $\bar{g}$ of $S$. Let $b$ be the automorphism of $A[G]=S \otimes T$ extending $\bar{g}$ and fixing the elements of $T$. Since $S$ and $T$ are right stable, $b(s t . x)=b(s t) . x$ for all $x \in G, s \in S$, and $t \in T$ because both are equal to $(b(s) \cdot x)(t . x)$. Hence $b$ is proper, so $b \in G$. Now $g b^{-1} \in S^{\prime}$ and $b \in T^{\prime}$. Hence $G=S^{\prime} . T^{\prime}$ (semi-direct) since $A[G]=S \otimes T$. Finally, it is evident that $S \subset A[G]^{S^{\prime}}$ and $T \subset A[G]^{T^{\prime}}$. Now $A[G]=S \otimes T$ and, as in the affine case, $A[G]=A[G]^{S^{\prime}} \otimes A[G]^{T^{\prime}}$ (cf. [H1, p. 71]). Hence $S=A[G]^{S^{\prime}}$ and $T=A[G]^{T^{\prime}}$. In particular, $S$ is a Hopf subalgebra of $A[G]$. Moreover, as in the affine case, $A[G]^{S^{\prime}}$ is Hopf-algebra isomorphic to $A\left[T^{\prime}\right]$, and $A[G]^{T^{\prime}}$ is algebra isomorphic to $A\left[S^{\prime}\right]$. This proves Lemma 2.1 .

Let $H(L)=C \otimes B$ and $B=H(L)^{R} \otimes V^{*}$ as in Theorem 1.1. Let $U$ be the fixer of $C \otimes H(L)^{R}$ in $G(L)$ (under left translations), let $T$ be the fixer of $B$ in $G(L)$, and let $S$ be the fixer of $C \otimes V^{*}$ in $G(L)$. Then we have the following result.

Theorem 2.2. $G(L)=U .(T \times S)$ (semi-direct) with the following properties:

(1) $U$ is the unipotent radical of $G(L)$ and $\operatorname{dim} U=\operatorname{dim} \operatorname{rad}(L)$.

(2) $T$ is a pro-toroid whose character group $X(T)$ is isomorphic to $P(L)$ and $A[G]^{T}$ is a normal basic subalgebra of $H(L)$. 
(3) $S$ is a simply connected semisimple algebraic group whose Lie algebra is isomorphic to a maximal semisimple sub Lie algebra of $L$.

Theorem 2.2 is an immediate consequence of Hochschild's result Theorem 1.1 in view of Lemma 2.1 and the following remarks. (i) $(H(L))_{S}=C \otimes B_{S}$ and $B_{S}=H(L)^{R}$, so $U$ is the fixer of $H(L)_{S}$. Hence $U$ is the unipotent radical of $G(L)[\mathbf{H}-\mathbf{M} 4$, p. 1134]. Since $A[U]$ is algebra isomorphic to $V^{*}$ whose (Krull) dimension is equal to $\operatorname{dim} \operatorname{rad}(L), \operatorname{dim} U=\operatorname{dim} \operatorname{rad}(L)$. (ii) $X(T) \approx Q(L)$ and $Q(L) \approx P(L)$ under the exponential map. Hence $X(T) \approx P(L)$. (iii) $A[S]$ is Hopf-algebra isomorphic to $H(L)^{R}$ which may be identified with $H(L / R)$. Hence $\mathcal{L}(S) \approx L / R$ as follows from the fact that a semisimple Lie algebra is universally algebraic, and $S$ is simply connected [H4, Thm. 3.1].

An affine Hopf subalgebra of $H(L)$ is called regular if it contains some (and hence every) normal basic subalgebra of $H(L)$. If $G$ is a pro-affine algebraic group, then $Q(G)$ is the intersection of the radical of $G$ with the reductive part of the center of $G$.

Theorem 2.3. Let $H$ be a connected affine algebraic group (over $F$ ), and let $i: L \rightarrow \mathcal{L}(H)$ be a Lie algebra homomorphism whose image is algebraically dense. Identify $A[H]$ with its canonical image in $H(L)$, and let $f: G(L) \rightarrow H$ be the restriction (surjective) morphism. Then the following are equivalent.

(1) $A[H]$ is a regular Hopf subalgebra of $H(L)$.

(2) $i$ is injective, $P(L) \subset A[H]$, and $H / \operatorname{rad}(H)$ is simply connected.

(3) $i$ is injective, $\operatorname{dim} H_{u}=\operatorname{dim} \operatorname{rad}(L)$, and $H / \operatorname{rad}(H)$ is simply connected.

(4) $i$ is injective, $\mathcal{L}(H)=i(L)+T$ (semi-direct) for every maximal toral subalgebra $T$ of the radical of $\mathcal{L}(H)$, and $H / \operatorname{rad}(H)$ is simply connected.

(5) $\operatorname{ker} f \subset Q(G(L))$. That is, ker $f$ is a reductive central algebraic subgroup of $G(L)$ contained in the radical of $G(L)$.

Proof. First we show that (1) implies (2). $A[H]$ is then a regular Hopf subalgebra of $H(L)$, so it contains a normal basic subalgebra $B$ of $H(L)$. Since $B$ separates the elements of $L$, it follows that $i: L \rightarrow \mathcal{L}(H)$ is injective. Since $P(L) \subset B$ [H3, p. 615], $P(L) \subset A[H]$. Moreover $B_{S}=H(L)^{R}$, so $H(L)^{R} \subset A[H]$. By restriction, this yields a surjective morphism $H \rightarrow S$ where $S$ is the algebraic group associated with the Hopf algebra $H(L)^{R}$. As in Theorem 2.2, $S$ is a simply connected semisimple algebraic group. Hence $H / \operatorname{rad}(H)$ is simply connected, so (1) implies (2). Now we show (2) 
implies (3). Let $U$ be the unipotent radical of $G(L)$ and let $f_{u}: U \rightarrow H_{u}$ be the restriction of $f$ to $U$ which is surjective because $f$ is surjective. We need to show that $f_{u}$ is an isomorphism. Since $P(L) \subset A[H]$, it follows that every additive (rational) character of $U$ induces an additive character of $H_{u}$ via the morphism $f_{u}$. But the additive characters of $U$ separate $U /[U, U]$. Hence $\operatorname{ker} f_{u} \subset[U, U]$. If $L^{\prime}$ is the Lie algebra of $G(L)$, then $\left[L^{\prime}, L^{\prime}\right] \subset L\left[\mathbf{H} 2\right.$, p. 521]. Consequently $\mathcal{L}\left(\operatorname{ker} f_{u}\right) \subset L \cap \operatorname{ker} f^{\circ}$. But this last is $\operatorname{ker} i$ and $i$ is injective. Hence $\operatorname{ker} f_{u}=(1)$. Thus $f_{u}: U \rightarrow H_{u}$ is an isomorphism. By Theorem 2.2, $\operatorname{dim} U=\operatorname{dim} \operatorname{rad}(L)$. Hence $\operatorname{dim} H_{u}=$ dim $\operatorname{rad}(L)$, so (2) implies (3). Let $R$ be a maximal reductive algebraic subgroup of $H$ containing $T$. Since $\mathcal{L}(H)=\mathcal{L}\left(H_{u}\right)+\mathcal{L}(R)$, it follows that $i(L)+T=i(L)+\mathcal{L}(R)$ is the Lie algebra of an algebraic subgroup of $H$. Hence $\mathcal{L}(H)=i(L)+T$ since $i(L)$ is algebraically dense in $\mathcal{L}(H)$. Moreover, $\operatorname{dim} H=\operatorname{dim} L+\operatorname{dim} T$ if and only if $\operatorname{dim} H_{u}=\operatorname{dim} \operatorname{rad}(L)$. Hence (3) is equivalent to (4).

We next show that (3) implies (5). Then we have $\operatorname{dim} U=\operatorname{dim} H_{u}=$ $\operatorname{dim} \operatorname{rad}(L)$, so that the surjective morphism $f_{u}: U \rightarrow H_{u}$ is an isomorphism. Hence ker $f$ is reductive. Put $G=G(L)$. To prove $[G$, ker $f]=(1)$, let $I$ be an affine Hopf subalgebra of $H(L)$ containing $A[H]$, and let $G_{I}$ be the restriction image of $G$ in the algebraic group $G(I)$ with Hopf algebra $I$ so that $G_{I}$ coincides with $G(I)$. Since $I$ contains $A[H], f$ induces a surjective morphism $f_{I}: G_{I} \rightarrow H$. Since $G$ is connected, so is $G_{I}$. Hence $\left[G_{I}\right.$, ker $\left.f_{I}\right]$ is a (connected) algebraic subgroup of $G_{I}$. Since $i$ is injective, $A[H]$, and hence $I$, separates the elements of $L$. Thus $L$ can be identified with its image in $\mathcal{L}\left(G_{I}\right)$ and, as such is algebraically dense in $\mathcal{L}\left(G_{I}\right)$. Hence $\mathcal{L}\left[G_{I}, \operatorname{ker} f_{I}\right]=(0)$, so $\left[G_{I}, \operatorname{ker} f_{I}\right]=(1)$ being connected. This shows that the restriction image in $G_{I}$ of the algebraic hull of $[G, \operatorname{ker} f]$ in $G$ is trivial for every affine Hopf subalgebra $I$ of $H(L)$ containing $A[H]$. But $H(L)$ is the union of such $I$ 's because every affine subalgebra of $H(L)$ is contained in an affine Hopf subalgebra of $H(L)$ [H5, p. 400]. Hence ker $f$ is central in $G(L)$ whenever $i: L \rightarrow \mathcal{L}(H)$ is injective. To prove $\operatorname{ker} f \subset \operatorname{rad}(G(L))$, let $G(L)=U(T \times S)$ be as in Theorem 2.2, so $U T$ is the radical of $G(L)$. Since $\mathcal{L}(S) \approx L / R$ and $i: L \rightarrow \mathcal{L}(H)$ is injective, then the restriction of $f^{\circ}$ to $\mathcal{L}(S)$ is also injective. But $H / \operatorname{rad}(H)$ is simply connected. Therefore ker $f \cap S=(1)$ and $\operatorname{rad}(H)$ is a semi-direct factor of $H$. Hence $\operatorname{ker} f \subset \operatorname{rad}(G(L))$. Thus (3) implies (5).

Finally, we show that (5) implies (1). As in Theorem 2.2, $G(L)=$ $U(T \times S)$ and $A[G(L)]^{T}$ is a normal basic subalgebra of $H(L)=A[G(L)]$. But ker $f \subset Q(G(L))$ which is contained in $T$. Hence $A[G(L)]^{T} \subset A[G(L)]^{\operatorname{ker} f}$. Moreover, this last coincides with $A[H]$ because $H$ can be identified with $G(L) /$ ker $f$. Hence $A[H]$ is a regular Hopf subalgebra of $H(L)$. This completes the proof of Theorem 2.3. 
The analogous affine (resp. pro-affine) characterizations for the regular hulls of complex analytic groups are given in [H-M3, Thms. 3.1 and 4.1] and [Ma1, Thm. 11] (resp. [Ma3, Thm. 5] and [N, Thm. 6]).

Theorem 2.4. Let $i: L \rightarrow \mathcal{L}(H)$ and $f: G(L) \rightarrow H$ be as in Theorem 2.3. Then the following are equivalent.

(1) There exists an affine covering $\hat{H} \rightarrow H$ which is an isomorphism on the radicals such that the canonical image of $A[\hat{H}]$ in $H(L)$ is regular.

(2) $i$ is injective and $P(L) \subset H(L)$.

(3) $i$ is injective and $\operatorname{dim} H_{u}=\operatorname{dim} \operatorname{rad}(L)$.

(4) $i$ is injective and $\mathcal{L}(H)=i(L)+T$ (semi-direct) for every maximal toral subalgebra $T$ of the radical of $\mathcal{L}(H)$.

(5) ker $f$ is a reductive central algebraic subgroup of $G(L)$.

The proof of Theorem 2.3 implies result in Theorem 2.4 in view of the following remarks. (i) Every affine covering $\hat{H} \rightarrow H$ induces a bijection between the additive (rational) characters of $\hat{H}$ and the additive characters of $H$. Moreover, every affine algebraic group $H$ has an affine covering $\alpha$ : $\hat{H} \rightarrow H$ which is an isomorphism on the radicals such that $\hat{H} / \operatorname{rad} \hat{H}$ is simply connected (for example, if $\tau: \operatorname{rad}(H) \rightarrow H$ is the injection morphism, then the last morphism of the universal $\tau$-extension $\operatorname{rad}(H) \rightarrow \hat{H} \rightarrow H$ given in Theorem 17 of $[\mathbf{N}]$, is the unique covering $\alpha$ ).

(ii) Let $f: G(L) \rightarrow H$ be as in Theorem 2.3. Then our covering morphism $\alpha: \hat{H} \rightarrow H$ above yields an algebraic hull $L \rightarrow \mathcal{L}(\hat{H})$ of $L$ whose associated canonical morphism $\hat{f}: G(L) \rightarrow \hat{H}$ satisfies $\alpha \circ \hat{f}=f$. In particular, ker $\hat{f} \subset Q(G(L))$ if and only if ker $f$ is a reductive central algebraic subgroup of $G(L)$.

\section{Definition 2.5.}

(i) An algebraic hull of $L$ is a connected affine algebraic group $H$ together with an injective morphism $i: L \rightarrow \mathcal{L}(H)$ with algebraically dense image.

(ii) An algebraic hull $i: L \rightarrow \mathcal{L}(H)$ of $L$ is called regular (resp. split) if it satisfies any the equivalent conditions given in Theorem 2.3 (resp. Theorem 2.4).

(iii) An algebraic hull $i: L \rightarrow \mathcal{L}(H)$ of $L$ is called reduced if $\operatorname{rad} H$ has unipotent center.

Corollary 2.5. Let $B(L)$ be the basic group of $L$.

(i) Then $B(L)$ coincides with $G(L) / Q(G(L))$.

(ii) If $i: L \rightarrow \mathcal{L}(H)$ is a reduced regular algebraic hull of $L$, then there 
exists an isomorphism $B(L) \rightarrow H$ whose differential agrees with $i$ on $L$.

(iii) If $i: L \rightarrow \mathcal{L}(H)$ is a regular algebraic hull of $L$. Then there exists a canonical surjective morphism $f: H \rightarrow B(L)$ such that $f^{0} \circ i$ is the identity map on L. Moreover, $\operatorname{ker} f=Q(H)$ so that $B(L)$ is canonically isomorphic with $H / Q(H)$.

Corollary 2.5 is an immediate consequence of Theorem 2.3 in view of the following remarks:

1. Let $G$ be a connected pro-affine algebraic group (over $F$ ). Then $Q(G)=$ (1) if and only if $\operatorname{rad}(G)$ has unipotent center. To see this, let $G=$ $G_{u} . R$ be a semi-direct decomposition of $G$ where $R$ is a maximal reductive algebraic subgroup of $G$ [H-M4, Thm. 3.2]. Then $R=T S$ where $T$ is the connected component of the identity element of the center of $R$, and $S$ is the algebraic hull of $[R, R]$ in $R$, so $G_{u} T$ is the radical of $G$ [H5, p. 413]. This implies that $Q(G)=Q(\operatorname{rad}(G))$. In particular, $Q(G)=(1)$ if and only if $\operatorname{rad}(G)$ has unipotent center.

2. Let $f: G \rightarrow H$ be a surjective morphism of connected pro-affine algebraic groups (over $F$ ). Then $f(Q(G)) \subset Q(H)$. Moreover, if ker $f$ is reductive, then $f(Q(G))=Q(H)$ as seen in the proof of Lemma 11 of [N]. (See also [Ma4, Prop. 6].)

Similarily, we have the following consequences of Theorem 2.3.

Corollary 2.6 [H6, Thm. 3.2]. $L$ is algebraically dense in $\mathcal{L}(B(L))$ and $\operatorname{rad} B(L)$ has unipotent center. If $H$ is any connected affine algebraic group satisfying these conditions, then there exists a (canonical) surjective morphism $B(L) \rightarrow H$ whose differential coincides with the identity map on $L$.

Remark. In the setting of Corollary 2.6, let $\rho: B(L) \rightarrow H$ be the canonical surjective morphism whose differential agrees with $i$ on $L$. Then $\operatorname{ker} \rho$ is in the center of $B(L)$ and $(\operatorname{ker} \rho)_{1}$ is a direct factor of $B(L)$ which is an algebraic vector group [R1, Prop. 2.4].

Corollary 2.7. Let $\phi: L_{1} \rightarrow L_{2}$ be a surjective morphism of Lie algebras. Then $\phi$ induces a surjective morphism $f: B\left(L_{1}\right) \rightarrow B\left(L_{2}\right)$ whose differential agrees with $\phi$ on $L_{1}$.

\section{Algebraic Lie algebras.}

For convenience, a connected algebraic group $G$ is called almost simply connected if its radical $R$ has unipotent center and $G / R$ is simply connected. Note that the basic group $B(L)$ is almost simply connected by Corollary 2.5. 
Theorem 3.1. Let $L$ be an algebraic Lie algebra, i.e., $L$ is the Lie algebra of some affine algebraic group. Then there exists a unique (up to isomorphism) almost simply connected affine algebraic group with Lie algebra L. Moreover, such an affine algebraic group is a direct factor of the basic group $B(L)$ of $L$. Furthermore, $B(L)$ has a direct factor $Z$ which is an algebraic vector group such that $\mathcal{L}(B(L))=L \oplus \mathcal{L}(Z)$ so that if $Z$ is any such direct factor of $B(L)$, then $B(L) / Z$ is almost simply connected with Lie algebra $L$.

Proof. Since $L$ is an algebraic Lie algebra, $L=\mathcal{L}(G)$ for some affine algebraic group $G$ which may be chosen to have unipotent center [H1, p. 250]. By Corollary 2.6 and the above remark, there exists a surjective morphism $\rho: B(L) \rightarrow G$ such that $(\operatorname{ker} \rho)_{1}$ is a direct factor of $B(L)$ which is an algebraic vector group. Put $Z=(\operatorname{ker} \rho)_{1}$. Then $\mathcal{L}(B(L))=L \bigoplus \mathcal{L}(Z)$, so $\mathcal{L}(B(L) / Z) \approx L$. Moreover $B(L)$, and hence, $B(L) / Z$ is almost simply connected by Corollary 2.5. Now let $G^{\prime}$ be any almost simply connected affine algebraic with Lie algebra $L$. Let $\rho^{\prime}: B(L) \rightarrow G^{\prime}$ and $Z^{\prime}=\left(\operatorname{ker} \rho^{\prime}\right)_{1}$ be defined in the same way as above. Then $B(L)=Z \times H=Z^{\prime} \times H^{\prime}$ for some algebraic subgroups $H$ and $H^{\prime}$ of $B(L)$. But $Z$ and $Z^{\prime}$ are algebraic vector groups of the same dimension. Hence $H$ is isomorphic to $H^{\prime}$ [R1, p. 290], so $H^{\prime}$ is isomorphic to $B(L) / Z$. On the other hand, the restriction of $\rho^{\prime}$ to $H^{\prime}$ is a covering morphism onto $G^{\prime}$. But $H^{\prime}$ and $G^{\prime}$ are almost simply connected (in particular their radicals are semi-direct factors). Hence our covering morphism is an isomorphism from $H^{\prime}$ to $G^{\prime}$. Thus $G^{\prime}$ is isomorphic to $B(L) / Z$. This completes the proof of Theorem 3.1.

Corollary 3.2 (cf. [H1, Thm. 2.2, p. 252], [H7, p. 10]). Let L be an algebraic Lie algebra. Then there exists a unique (up isomorphism) connected affine algebraic group with unipotent center, and with Lie algebra L. Moreover, if $H=B(L) / Z$ is the unique (up to isomorphism) almost simply connected affine algebraic group with Lie algebra L, as in Theorem 3.1, and $C(H)$ is the (finite) maximal reductive central algebraic subgroup of $H$, then $H / C(H)$ is a connected affine algebraic group with unipotent center, and with Lie algebra $L$

Proof. Let $H=R . S$ (semi-direct) where $R=\operatorname{rad}(H)$ and $S$ is a maximal semisimple algebraic subgroup of $H$. Since $H=B(L) / Z$ is almost simply connected, $C(H) \subset S$. Let $S_{0}$ be the center of $S$. Since $S_{0}$ is a finite algebraic subgroup of $H$, and $H$ is connected, it follows that $S_{0}$ is in the center of $H$ (for example, $\left[H, S_{0}\right]$ is connected with trivial Lie algebra). Hence $C(H)=S_{0}$. Now $H=R . S$ (semi-direct), $R$ has unipotent center, and $\mathcal{L}(H)=L$. Hence $H / C(H)$ has unipotent center with Lie algebra $L$. Now let $G$ be any connected affine algebraic group with unipotent center 
whose Lie algebra is $L$. As in the proof of Theorem 3.1, we have a surjective morphism $\rho^{\prime}: B(L) \rightarrow G$ where $B(L)=Z^{\prime} \times H^{\prime}$ and $Z^{\prime}=\left(\operatorname{ker} \rho^{\prime}\right)_{1}$. Since $G$ has unipotent center, it follows that the kernel of the restriction of $\rho^{\prime}$ to $H^{\prime}$ is $C\left(H^{\prime}\right)$. Hence $G$ is isomorphic to $H^{\prime} / C\left(H^{\prime}\right)$. But $H$ is isomorphic to $H^{\prime}$ as seen in the proof of Theorem 3.1. Hence $G$ is isomorphic to $H / C(H)$.

\section{Lie algebras with the same Hopf algebra.}

We shall need the fact every connected affine algebraic group over $F$ has a universal (simply connected) pro-finite covering [H5, Thm. 5.1]. Moreover, a pro-toroid $T$ is simply connected if and only if its character group $X(T)$ is a rational vector space $[\mathbf{P}$, p. 220]. Note that, if $K$ is a normal algebraic subgroup of a pro-affine algebraic group $G$, then $G$ is simply connected if and only if $K$ and $G / K$ are simply connected [H5, Thm. 5.2].

The following general result is contained in the proof of Theorem 6 of [Ma3] in which Magid shows that, over $\mathbb{C}, G(L) / Q(G(L))$ determines $G(L)$ up to isomorphism.

Theorem 4.1. Let $G$ be a connected pro-affine algebraic group over $F$ such that $G=G_{u} .(T \times S)$ (semi-direct) where $G_{u}$ is affine, $T$ is a pro-toroid whose character group $X(T)$ is a rational vector space, and $S$ is a simply connected semisimple affine algebraic group (so $G$ is simply connected). Let $f: G \rightarrow H$ be a surjective morphism onto a connected affine algebraic group $H$ such that $(\operatorname{ker} f)_{1}$ is a central pro-toroid of $G$. Then $H$ determines $G$ up to isomorphism. Specifically, if $\hat{H}$ is the universal pro-finite covering of $H$, and $D$ is the pro-toroid with character group $\operatorname{Hom}(H, F)$, then $G$ is isomorphic to $\hat{H} \times D$.

Corollary 4.2. Let $i: L \rightarrow \mathcal{L}(H)$ be a split hull of $L$ (see Def. 2.5). Then the affine algebraic group $H$ determines the Hopf algebra $H(L)$ up to isomorphism. If $\hat{H}$ is the univeral (pro-finite) covering of $H$ [H5, Thm. 5.1.] and $D$ is the pro-toroid whose character group is $\operatorname{Hom}(H, F)$, then $H(L)$ is isomorphic to the Hopf algebra of polynomial functions of the pro-affine algebraic group $\hat{H} \times D$.

Proof. Combine Theorems 4.1 and 2.4.

Corollary 4.3. The basic group $B(L)$ of $L$, or its Lie algebra, determines the Hopf algebra $H(L)$ up to isomorphism. In particular, two Lie algebras have isomorphic Hopf algebras of representative functions if and only if they have isomorphic basic groups, or equivalently they have isomorphic basic Lie algebras (Lie algebras of basic groups).

Proof. Combine Corollaries 4.2 and 2.5 with Theorem 3.1. 
The next Theorem gives a simple construction of the basic Lie algebra of $L$ directly from the adjoint representation of $L$.

Theorem 4.4. Let ad $: L \rightarrow$ DerL be the adjoint representation of $L$. Let $\operatorname{ad}(L)^{+}$be the algebraic hull of ad $(L)$ in DerL, and let $T$ be a maximal toral subalgebra of the radical of ad $(L)^{+}$. Then the semi-direct Lie algebra $L^{\sharp}=L+T$ (with the adjoint action of $T$ on $L$ ) is isomorphic to the Lie algebra of the basic group $L$ under an isomorphism which is the identity on $L$ and mapping $T$ onto the Lie algebra of a maximal toroid of the radical of the basic group of $L$.

Proof. Since $L$ is an ideal of $\mathcal{L}(B(L))$, the adjoint representation of $\mathcal{L}(B(L))$ induces a Lie algebra homomorphism $\alpha: \mathcal{L}(B(L)) \rightarrow \operatorname{Der} L$ which agrees with $a d$ on $L$. Let $D$ be a maximal toral subalgebra of $\alpha^{-1}(T)$. Then $\alpha(D)=T$. Since $T$ is in the radical $\alpha(L)^{+}$, it follows that $D$ is in the radical of $\mathcal{L}(B(L))$. Since $B(L)$ has no central tori, it follows that the restriction of $\alpha$ to $D$ is an isomorphism onto $T$. Moreover, $D$ is a maximal toral subalgebra of the radical of $\mathcal{L}(B(L))$ because $T$ is a maximal toral subalgebra of the radical of $a d(L)^{+}$. Hence $\mathcal{L}(B(L))=L+D$ (semi-direct) by Theorem 2.3. Since $\alpha$ maps $D$ isomorphically onto $T \subset \operatorname{Der} L$, it follows that there exists an isomorphism from the Lie algebra $L^{\sharp}=L+T$ (with the adjoint action of $T$ on $L$ ) onto $\mathcal{L}(B(L))$ which is the identity on $L$ and mapping $T$ onto $D$. This completes the proof of Theorem 4.4.

Our next goal is to construct all Lie algebras whose Hopf algebras are isomorphic to $H(L)$. Let $D$ be a maximal toral subalgebra of the radical of $\mathcal{L}(B(L))$, and put $A=\mathcal{L}\left(B(L)_{u}\right)+[L, L]$. Since $\mathcal{L}(B(L))=L+D$ (semi-direct), it follows that $\mathcal{L}(B(L))=A+D$ (semi-direct). This last decomposition yields a projection morphism $\mathcal{L}(B(L)) \rightarrow D$ which is called the rational projection of $\mathcal{L}(B(L))$ onto $D$. Since $L$ is algebraically dense in $\mathcal{L}(B(L))$, then so is its rational projection image in $D$.

Similarly, let $L^{\sharp}=L+T$ (semi-direct) as in Theorem 4.4, and put $\mathbf{A}(L)=$ $\operatorname{nil}\left(L^{\sharp}\right)+[L, L]$. Then the isomorphism of $L^{\sharp}$ with $\mathcal{L}(B(L))$ given in Theorem 4.4 implies that $L^{\sharp}=\mathbf{A}(L)+T$ (semi-direct). This yields a projection morphism $L^{\sharp} \rightarrow T$ which is called the rational projection of $L^{\sharp}$ on $T$. The proof of Theorem 4.4 shows that this rational projection maps $L$ onto a dense subalgebra of $T=\mathcal{L}\left(T_{g}\right)$ where $T_{g}$ is the toroid of Aut $L$ with Lie algebra $T$. Hence Theorem 4.4 with Corollaries 4.3 and 2.5 imply the following result.

Theorem 4.5. Let ad $: L \rightarrow \operatorname{Der} L$ and $L^{\sharp}=L+T$ (semi-direct) be as in Theorem 4.4. Let $\gamma: L^{\sharp} \rightarrow T$ be the rational projection of $L^{\sharp}$ onto $T$. Let $\left\{L_{i}\right\}$ be the ideals of $L^{\sharp}$ such that, for each $i, L^{\sharp}=L_{i}+T$ (semi-direct) and $\gamma\left(L_{i}\right)$ is algebraically dense in $T=\mathcal{L}\left(T_{g}\right)$ where $T_{g}$ is the toroid of AutL 
whose Lie algebra is $T$. Then $\left\{L_{i}\right\}$ is the set of all Lie algebras whose Hopf algebras are isomorphic to $H(L)$.

\section{Hopf algebras of Lie algebras.}

First we characterize those algebraic groups which are basic groups of Lie algebras. The proof of Theorem 5.1 below is a modification of the proof for bottom groups in the complex case given by Magid in Theorem 13 of [Ma3].

Theorem 5.1. Let $G$ be an affine algebraic group. Then $G$ is the basic group of some Lie algebra if and only if $G$ is almost simply connected with the property that either $G_{u}=\operatorname{rad}(G)$ or $G$ has a non-trivial additive rational character.

Proof. Suppose $G=B(L)$ for some Lie algebra $L$. Then Corollary 2.5 shows that $B(L)$ is almost simply connected. Moreover, if $B(L)$ has no non-trivial additive characters, then the same is true for $G(L)$, so $P(L)=(0)$. Hence $G(L)_{u}=\operatorname{rad}(G(L))$ by Theorem 2.2, so $(B(L))_{u}=\operatorname{rad}(B(L))$. Conversely, suppose $G$ is almost simply connected with the property that either $G_{u}=$ $\operatorname{rad}(G)$ or $G$ has a non-trivial additive character. If $G_{u}=\operatorname{rad}(G)$, then $G$ is the basic group of $L=\mathcal{L}(G)$ by Corollary 2.5, so we assume that $G$ has a nontrivial additive character $f$. Let $T=\left(F^{*}\right)^{n}$ be a maximal toroid of $\operatorname{rad}(G)$, and choose $\alpha_{1} \ldots \alpha_{n} \in F$ be linearly independent over the field of rationals. Put $A=\mathcal{L}\left(G_{u}\right)+[\mathcal{L}(G), \mathcal{L}(G)]$, and let $\phi: A \rightarrow \mathcal{L}(T)$ be the map given by $\phi(a)=\left(\alpha_{1} f^{0}(a), \ldots, \alpha_{n} f^{0}(a)\right)$. Then the set $L$ of all elements $a+\phi(a)$ with $a \in A$ is a sub Lie algebra of $\mathcal{L}(G)$ because it contains $[\mathcal{L}(G), \mathcal{L}(G)]$. Since $\operatorname{rad}(\mathcal{L}(G))=\mathcal{L}\left(G_{u}\right)+\mathcal{L}(T)$, it follows that $L$ is algebraically dense in $\mathcal{L}(G)$. Moreover, $\operatorname{rad}(L)$ and $\mathcal{L}\left(G_{u}\right)$ have the same dimension. Hence $G$ is the basic group $L$ by Corollary 2.5. This proves Theorem 5.1.

Finally, we characterize the Hopf algebras of Lie algebras. Recall that if $B$ is an affine algebra containing an affine algebra $A$, then $B$ is called an affine unramified extension of $A$ if $B$ is finitely-genrated as an $A$-module, and for every $B$-module $M$, the only $A$-linear derivation of $B$ into $M$ is the zero map. We shall use Theorem 4.1 of $[\mathbf{H} 4]$ which says that if $G$ is a connected affine algebraic group over $F$, then $G$ is simply connected if and only if $\mathbf{A}[H]$ has no proper affine extensions.

Theorem 5.2 (cf. [R2, Thm. 2.1]). Let $A$ be a commutative Hopf algebra over $F$. Then $A$ is isomorphic to some Hopf algebra $H(L)$, where $L$ is a finite-dimensional Lie algebra, if and only if A satisfies the following properties:

(1) $A$ is an integral domain; 
(2) there is a group isomorphism from the additive group $P$ of the primitive elements of $A$ onto the multiplicative group $Q$ of the group-like elements of $A$;

(3) there exists a right stable affine subalgebra $B$ of $A$ such that $A=$ $B \otimes F[Q]$ and $B$ has no proper affine unramified extensions.

Proof. If $A=H(L)$ for some Lie algebra $L$, then Theorems 1.1 and 2.2 imply that $A$ satisfies (1)-(3). In fact the normal basic sub algebra $B=A[G]^{T}$ in Theorem 2.2 has no proper unramified extensions because $B$ is isomorphic to the algebra $A[U S]$ and $U S$ is a simply connected affine algebraic group. Conversely, suppose $A$ satisfies (1)-(3), and let $A=A[G]$ where $G$ is the proaffine algebraic group with Hopf algebra $A$. Then Lemma 2.1 implies that $G=H . T$ (semi-direct) where $H$ is a simply connected affine algebraic group whose algebra is isomorphic to $B$ and $T$ is a pro-toroid whose character group is isomorphic to $Q$, and hence $P$. In fact $T$ is simply connected because its character group $P$ is a rational vector space. Since $H$ is simply connected and affine, $H=U S$ (semi-direct) where $U=H_{u}$ and $S$ is a (maximal) simply connected semisimple affine algebraic group of $H$ [H4, Thm. 2.3]. Hence $G=U S . T$ (semi-direct). Now $U$ is evidently normalized by $T$, so $U=G_{u}$.

Let $R$ be a maximal reductive algebraic subgroup of $G$ containing $S$, and let $T^{\prime}$ be the connected component of the centralizer of $S$ in $R$. Then $R=S . T^{\prime}$ where $S \cap T^{\prime}$ is finite as in [H5, p. 414] or in [N, Lemma 20]. Since $G=U R$ [H-M2] and $G / U S$ is simply connected being isomorphic to $T$, it follows that $G=U \cdot\left(S \times T^{\prime}\right)$. Moreover, $X\left(T^{\prime}\right)$ is isomorphic to $X(T)$, so $X\left(T^{\prime}\right)$ is isomorphic to $P=\operatorname{Hom}(G, F)$. But $G=U$. $\left(S \times T^{\prime}\right)$. Hence we either have $G_{u}=\operatorname{rad}(G)$ (i.e., $\left.T^{\prime}=(1)\right)$ or $G$ has a non-trivial additive rational character. Consequently, by Theorem 5.1, $G / Q(G)$ is the basic group of some Lie algebra $L$. By Corollary 4.2 and Theorem 4.1, $G(L)$ is then isomorphic to $G$ (as pro-affine algebraic groups) because both groups are isomorphic to $(G / Q(G))^{*} \times D$ where $(G / Q(G))^{*}$ is the universal pro-finite covering of $G / Q(G)$ and $D$ is the pro-toroid with character group $\operatorname{Hom}(G / Q(G), F)$. Hence $H(L)$ and $A[G]=A$ are isomorphic Hopf algebras. This proves Theorem 5.2.

The above proof shows that if $A=A[G]$ is a commutative Hopf algebra satisfying conditions (1)-(3) of Theorem 5.2, then the Lie algebra $L$ constructed in the proof of Theorem 5.1 with $G / Q(G)$ as a basic group has the property that $A$ is isomorphic to $H(L)$. 


\section{References}

[G] W. Giles, On the algebra of representative functions of a Lie algebra, Trans. Amer. Math. Soc., 109 (1963), 101-120.

[H1] G. Hochschild, Basic Theory of Algebraic Groups and Lie Algebras, Springer Verlag, GTM 75, 1981.

[H2] , Algebraic Lie algebras and representative functions, Illinois J. Math., 3 (1959), 499-523.

[H3] Algebraic Lie algebras and representative functions, Supplement, Illinois J. Math., 4(4) (1960), 609-618.

[H4] Algebraic groups and Hopf Algebras, Illinois J. Math., 14 (1970), 52-65.

[H5] _ Coverings of pro-affine algebraic groups, Pacific J. Math., 35 (1970), 399415.

[H6] Lie algebra cohomology and affine algebraic groups, Illinois J. Math., 18 (1974), 170-176.

[H7] Note on algebraic Lie algebras, Proc. Amer. Math. Soc., 29 (1971), 10-16.

[H-M1] G. Hochschild and G.D. Mostow, Representations and representative functions of Lie groups, Ann. of Math., 66 (1957), 495-542.

[H-M2] On the algebra of representative functions of an analytic group, Amer. J. Math., 83 (1961), 111-136.

[H-M3] - On the algebra of representative functions of an analytic group II, Amer. J. Math., 86 (1964), 869-887.

[H-M4] _ Pro-affine algebraic groups, Amer. J. Math., 91 (1969), 1127-1140.

[H-M5] Complex analytic groups and Hopf algebras, Amer. J. Math., 91 (1969), 1141-1151.

[L] A. Lubotzky, Tannaka duality for discrete groups, Amer. J. Math., 102 (1980), 663-689.

[Ma1] A.Magid, Analytic subgroups of affine algebraic groups, II, Pacific J. Math., 86 (1980), 145-154.

[Ma2] , Representative functions on simply connected solvable groups, Amer. J. Math., 102 (1980), 303-319.

[Ma3] _ Lie algebras with the same modules, Illinois J. Math., 25 (1981), 611-621.

[Ma4] , Analytic Representations of polycyclic groups, Journal of Algebra, 74 (1982), 149-158.

[Ma5] - Module categories of analytic groups, Cambridge University Press, 1982.

[Mi] F. Minbashian, Pro-affine algebraic groups, Amer. J. Math., 95 (1973), 174-192.

[N] N. Nahlus, Representative functions on complex analytic groups, Amer. J. Math., 116 (1994), 621-636.

[P] B. Peterson, Extensions of pro-affine algebraic groups, Pacific J. Math., 77 (1978), 189-231.

[R1] J.H. Reinoehl, Lie algebras and affine algebraic groups, Pacific J. Math., 86(2) (1980), 287-300.

[R2] _ Lie algebras and Hopf algebras, Pacific J. Math., 93 (1981), 181-192. 
[Ry] J.Ryan, Extensions of representations of Lie algebras, Pacific J. Math., 127 (1987), 173-186.

[S] M. Sweedler, Hopf Algebras, Benjamin, 1969.

Received March 16, 1994 and revised February 2, 1995.

AMERICAN UNIVERSITY OF BeIRUT

BeIRUt, LEBANON

E-mail address: nahlus@layla.aub.edu.lb 